\title{
A Need for Tetanus Vaccination Before Restoration Activities in Fukushima, Japan
}

\author{
Tomohiro Morita, MD; Masaharu Tsubokura, MD; Tetsuya Tanimoto, MD; \\ Tsuyoshi Nemoto, MD; Yukio Kanazawa, MD
}

$\mathrm{T}$ etanus is an infectious disease caused by exposure of wounds to spores of Clostridium tetani. The disease is fatal but preventable through vaccination. In Japan, tetanus vaccinations have been included in the routine national immunization program for children only since $1968 .{ }^{1}$ Thus, many of those who were born before 1968 are unimmunized against tetanus. Indeed, of 666 tetanus patients reported from 2006 to 2011 in Japan, 89\% (596/666) were over 50 years of age according to the National Institute of Infectious Diseases. ${ }^{2}$

In the Minamisoma Municipal General Hospital, located $25 \mathrm{~km}$ north of the Fukushima Daiichi Nuclear Power Plant (FNPP), we treated a 55-yearold man for a nail wound in November 2013 (Figure 1), 3 years after the FNPP accident. He sustained the nail puncture wound in his right foot while engaged in a volunteer activity of cleaning up housing wreckage in a restricted area in Fukushima. He was treated with antibiotics after irrigation and went through an uneventful clinical course. Because he had no knowledge of tetanus and had never received a tetanus vaccination, similar to other Japanese citizens of the same age, we informed him about the risk of tetanus and administered a complete course of tetanus vaccinations.

In March 2011 the Fukushima prefecture in Japan was damaged by the triple disaster of earthquake, tsunami, and the FNPP accident. The Japanese government designated restricted areas around the FNPP after the plant accident. After the decrease in the environmental radiation dose, residents or volunteers have been allowed to enter with time restrictions since April 16, 2012.

This restriction has delayed restoration of the areas. In Minamisoma, the city closest to the FNPP, cleanup activities have suffered from shortages of workers. Thus, the city remains covered with rubble and sludge (Figure 2). ${ }^{3}$ Cleanup workers or residents are at risk of injuries from the wreckage. However, no informational campaign on tetanus for those born before 1968 has been held in Minamisoma city like in other areas in Japan. In addition, the decreased medical resources in restricted areas may impede access to medical treatment. In the main 5 hospitals of Minamisoma City within $30 \mathrm{~km}$ of the FNPP, the number of nurses has decreased from 455 to 315 in 2 years. Therefore, 70 of 250 hospital beds are inactive in our hospital. Similarly, 12 of 39 clinics in the city are closed. The reduced availability of medical resources can delay the treatment of injuries leading to wound infections including tetanus. Thus, the people in Minamisoma City are at risk of tetanus. In fact, 2 cases of tetanus related to injuries with wreckage from the disasters were reported in peripheral cities in $2011,{ }^{4}$ although we have no reports in Minamisoma City as of February 2014.

This case illuminates the increased risk of tetanus infection for unvaccinated adults after a disaster like the FNPP accident in Japan. Preventive strategies against tetanus and educational campaigns on vaccination for unvaccinated workers look promising to reduce tetanus infection risk when the injury incident rate rises by a disaster. ${ }^{5}$

\section{FIGURE 1}

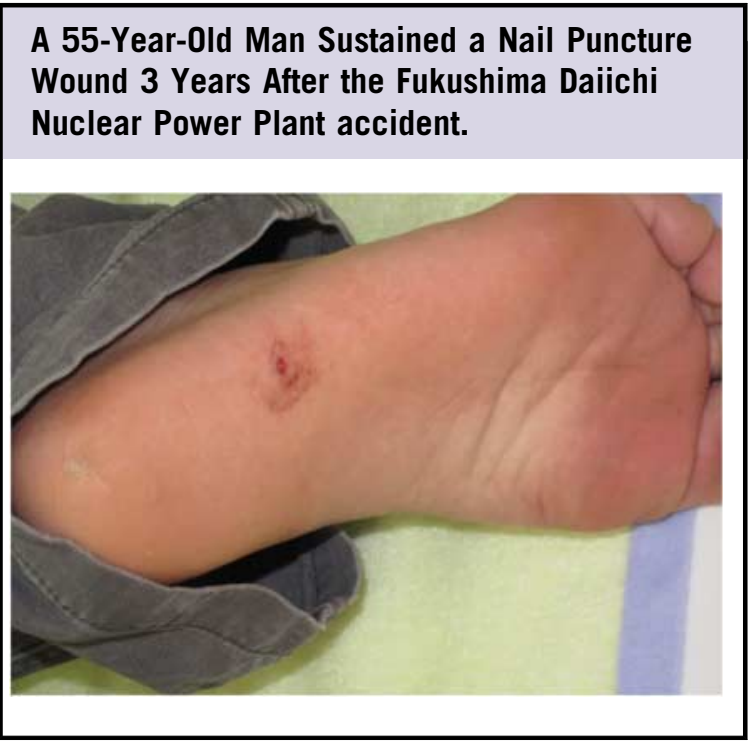


FIGURE 2

\section{Minamisoma, the City Closest to the Fukushima Daiichi Nuclear Power Plant, Remains Covered With Debris.}

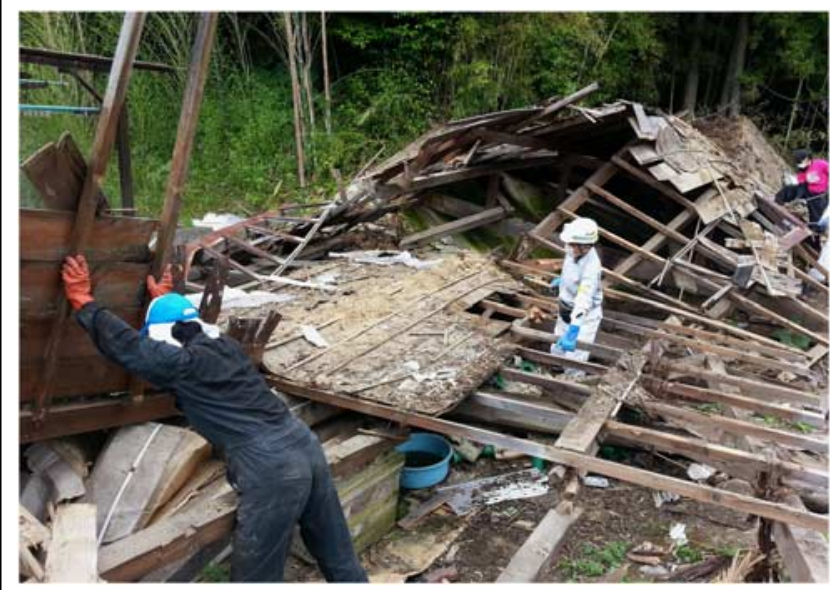

\section{About the Authors}

Postgraduate Medical Education Department, Kameda Medical Center, Kamogawa, Chiba, Japan (Dr Morita); Departments of Internal Medicine (Drs Morita and Tsubokura), Home Health Care (Dr Nemoto), Gastroenterology (Dr Kanazawa), Minamisoma Municipal General Hospital, Minamisoma, Fukushima, Japan; and Department of Internal Medicine, Jyoban Hospital of Tokiwakai Group, Fukushima, Japan (Dr Tanimoto).

Correspondence and reprint requests to Tomohiro Morita, MD, Postgraduate Medical Education Department, Kameda Medical Center, Kamogawa, Chiba, Japan 929 Higashi-Cho, Kamogawa-shi, Chiba 296-8602, Japan (e-mail: t.morita526@gmail.com).

Published online: November 7, 2014.

\section{REFERENCES}

1. Nakano T. Japanese vaccinations and practices, with particular attention to polio and pertussis. Travel Med Infect Dis. 2011;9:169-175.

2. The National Institute of Infectious Diseases Web site. http://www.nih.go. jp/niid/en/. Accessed February 11, 2014.

3. Tsubokura M, Kato S, Nihei M, et al. Limited internal radiation exposure associated with resettlements to a radiation-contaminated homeland after the Fukushima Daiichi nuclear disaster. PLoS One. 2013;8:e81909.

4. Aoyagi T, Yamada M, Kunishima H, et al. Characteristics of infectious diseases in hospitalized patients during the early phase after the 2011 great East Japan earthquake: pneumonia as a significant reason for hospital care. Chest. 2013;143:349-356.

5. Connolly. MA. Communicable Disease Control in Emergencies: A Field Manual. Geneva: World Health Organization; 2005. 